International Journal of Pure and Applied Mathematics

Volume 106 No. 2 2016, 415-427

ISSN: 1311-8080 (printed version); ISSN: 1314-3395 (on-line version)

url: http://www.ijpam.eu

doi: 10.12732/ijpam.v106i2.6

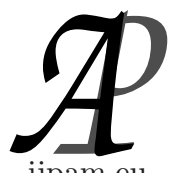

\title{
THE ATOM BOND CONNECTIVITY INDEX OF CERTAIN GRAPHS
}

\author{
Mohanad A. Mohammed ${ }^{1}$, K.A. Atan ${ }^{2}$, A.M. Khalaf ${ }^{3}$, \\ M. Rushdan ${ }^{4}$, R. Hasni ${ }^{5}$ \\ 1,3 Department of Mathematics \\ Faculty of Computer Science and Mathematics \\ University Of Kufa, Najaf, IRAQ \\ 1,2,4 Institute for Mathematical Research \\ Universiti Putra Malaysia \\ 43400, Serdang, Selangor, MALAYSIA \\ ${ }^{5}$ Department of Mathematics \\ Faculty of Science and Technology \\ University Malaysia Terengganu \\ 21030, UMT Terengganu, MALAYSIA
}

\begin{abstract}
The atom bond connectivity $(\mathrm{ABC})$ index provides a good model for the stability of linear and branched alkanes as well as the strain energy of cycloalkanes, which is defined as $A B C=\sum_{u v \in E(G)} \sqrt{\frac{d_{u}+d_{v}-2}{d_{u} d_{v}}}$, we denotes $d_{u}$ the degree of vertex $\mathrm{u}$ in $\mathrm{G}$. In this paper, we study the general formula for $\mathrm{ABC}$ index of certain graphs and vertex gluing of graphs.
\end{abstract}

AMS Subject Classification: $97 \mathrm{~K} 30$

Key Words: ABC Index, $K_{4}$-homeomorphism, complete bipartite, k-bridge graph and vertex gluing

\section{Introduction}

Topological indices are useful tools for modeling physical and chemical prop-

Received: August 25, 2015

Published: February 10, 2016

${ }^{\S}$ Correspondence author (c) 2016 Academic Publications, Ltd.

url: www.acadpubl.eu 
erties of molecules, for design of pharmacologically active compounds, for recognizing environmentally hazardous materials, etc. [7]. There are many publications on the topological indices; see [9-15]. One of the most important topological indices is the Randić index which is aimed at use in the modelling of the branching of the carbon-atom skeleton of alkanes, and was introduced by Randić [8].

In order to take this into account but at the same time to keep the spirit of the Randić index, Ernesto Estrada et al. proposed a new index, nowadays known as the atom-bond connectivity (ABC) index, and it has many chemical applications [2]. This index is defined as follows:

$$
A B C=\sum_{u v \in E(G)} \sqrt{\frac{d_{u}+d_{v}-2}{d_{u} d_{v}}} .
$$

The ABC index has proven to be a valuable predictive index in the study of the heat of formation in alkanes [1, 2]. In 2013, Wenshui Lin et al. [6], considered non-trivial connected simple graphs only. Such a graph will be denoted by $\mathrm{G}=(\mathrm{V}, \mathrm{E})$, where $V=\left\{v_{1}, \ldots, v_{n-1}, v_{n}\right\}$ and $\mathrm{E}=\mathrm{E}(\mathrm{G})$ are the vertex set and edge set of $\mathrm{G}$, respectively. If $v_{i} v_{j} \in E$, then $G-v_{i} v_{j}$ will denote the graph obtained from $\mathrm{G}$ by deleting the edge $v_{i} v_{j}$. If $v_{i} v_{j} \notin E$, then $G-v_{i} v_{j}$ will denote the graph obtained from $\mathrm{G}$ by adding the edge $v_{i} v_{j}$. When examining a topological index, one of the fundamental questions that needs to be answered is for which graphs this index assumes minimal and maximal values and what are these extremal values. In the case of the ABC index, finding the tree for which this index is maximal was relatively easy [3], it is the star. Eventually, also the trees with second-maximal, third-maximal, and so forth ABC index were determined [4]. Chen and Guo, [5] have shown that by deleting an edge from any graph, the ABC index decreases. This result implies that among all n-vertex graphs, the complete graph $K_{n}$ has maximal ABC value. Further, among all connected n-vertex graphs, minimal ABC is achieved by some tree.

Thus the n-vertex trees with minimal ABC index are also the n-vertex connected graphs with minimal ABC index. But the problem of characterizing the n-vertex trees with minimal ABC index turned out to be much more difficult, and a complete solution of this problem is not known. In this paper, we investigate the ABC index of some special graphs as well as a vertex gluing of graphs by finding general formulas. 


\section{Basic Definition and Known Results}

A $K_{4}$-homeomorphic graph or simply $K_{4}$-homeomorph, denoted by $K_{4}$ (a, b, c, $\mathrm{d}$, e, f ), is the graph obtained when the six edges of a complete graph with four vertices $\left(K_{4}\right)$ are subdivided into a, b, c, d, e, f segments, respectively. Each subdivided edge is called a path and its length is the number of resulting segments (see Fig.1).

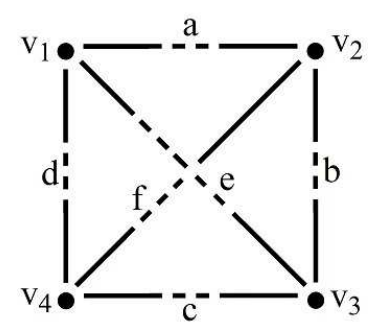

Figure 1: $K_{4}$-homeomorphic graph

A complete bipartite graph is a simple bipartite graph with partite sets $V_{1}$ and $V_{2}$ where every vertex in $V_{1}$ is adjacent with all the vertices in $V_{2}$. If $\left|V_{1}\right|=n$ and $\left|V_{2}\right|=m$, then such complete bipartite graph is denoted by $K_{n, m}$ (or $K(n, m)$ ). So $K_{n, m}$ has order $\mathrm{n}+\mathrm{m}$ and size $\mathrm{nm}$. A tree $K_{1, m}$ is also called a star (see Fig.2).

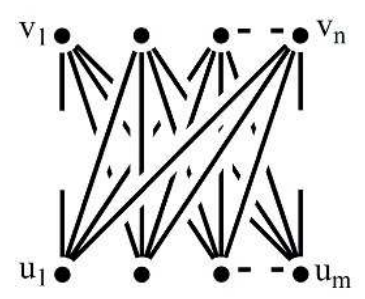

Figure 2: A complete bipartite $K_{n, m}$

A graph consisting of s paths joining two vertices is called an r-bridge graph, which is denoted by $\mathrm{Q}\left(a_{1}, \ldots, a_{r}\right)$, where $a_{1}, \ldots, a_{r}$, are the lengths of $\mathrm{r}$ paths. Clearly an r-bridge graph is a generalized polygon tree (see Fig.3).

A web graph $\operatorname{Web}(n, m)$ is the graph obtained from the Cartesian product of the cycle $C_{n}$ and the path $P_{m}$ (see Fig. 4). 


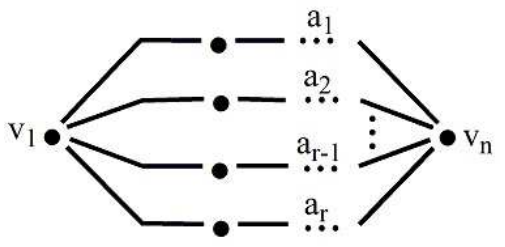

Figure 3: r-bridge graph

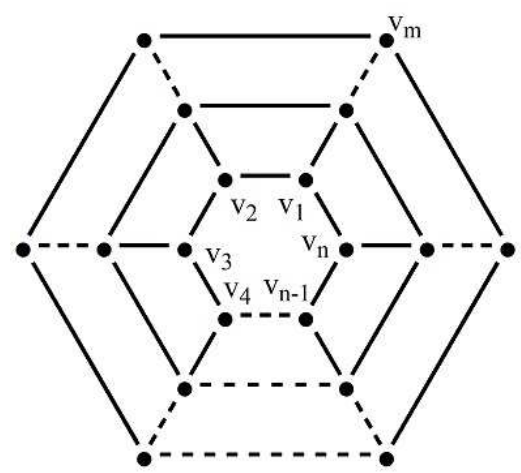

Figure 4: A web graph $\operatorname{Web}(n, m)$

We first give some examples of the ABC index for some simple graphs. Let $P_{n}, C_{n}, K_{n}$ and $S_{n}$ be the path, cycle, complete and star graphs respectively with $n$ vertices [16].

Lemma 2.1. Consider the complete graph $K_{n}$ of order $n$. The atom bond connectivity index of this graph is computed as follow:

$$
A B C\left(C_{n}\right)=\frac{1}{2} n \sqrt{2(n-2)}
$$

Lemma 2.2. Suppose $C_{n}$ is a cycle of length $n$ labeled by $1,2, \ldots, n$. Then the atom bond connectivity $(A B C)$ index of this cycle is

$$
A B C\left(C_{n}\right)=\frac{\sqrt{2}}{2} n .
$$


Lemma 2.3. If $S_{n}$ is the star on $n$ vertices, then

$$
A B C\left(S_{n}\right)=\sqrt{(n-1)(n-2)}
$$

Lemma 2.4. If $P_{n}$ is the Path on $n$ vertices, then

$$
A B C\left(P_{n}\right)=\frac{\sqrt{2}}{2}(n-1) .
$$

\section{The ABC Index of Some Special Graphs}

In this section, we obtain the general formulas for some special graphs.

Theorem 3.1. Let $a, b, c, d, e, f$ be positive integers. The atom bond connectivity index of a $K_{4}$-homeomorphism graph denoted by $K_{4}(a, b, c, d, e, f)$ are as follows:

1. If a or/and $b$ or/and $c$ or/and $d$ or/and e or/and $f=1$, then the $A B C$ index to any one of them is $\frac{2}{3}$.

2. If a or/and $b$ or/and $c$ or/and $d$ or/and e or/and $f \neq 1$, then the $A B C$ index to any one of them is (the number of edges) $\frac{1}{\sqrt{2}}$.

Proof. 1. If a or/and b or/and $\mathrm{c}$ or/and $\mathrm{d}$ or/and e or/and $f=1$ then any one of them has one edge and two vertices and the same degree three. Thus, the $\mathrm{ABC}$ index to any one of them will be $\frac{2}{3}$.

2. If $\mathrm{a}$ or/and $\mathrm{b}$ or/and $\mathrm{c}$ or/and $\mathrm{d}$ or/and e or/and $f \neq 1$ than, any one of them has two or more edges and each of them has two vertices at least one of the vertices of degree two. Thus, the ABC index to any one of them is (the number of edges) $\frac{1}{\sqrt{2}}$.

Example 3.2. Let $a, b, c, d, e, f$ be positive integers. The atom bond connectivity index of a $K_{4}$-homeomorphism graph denoted by $K_{4}(\mathrm{a}, \mathrm{b}, \mathrm{c}, \mathrm{d}, \mathrm{e}, \mathrm{f})$ is

$$
A B C\left(K_{4}(a, b, c, d, e, f)\right)=
$$




$$
\left\{\begin{array}{llc}
(a+b+c+d+e+f) \sqrt{\frac{1}{2}} ; & \text { if } & a, b, c, d, e, f \neq 1 \\
4 ; & \text { if } & a=b=c=d=e=f=1 \\
2+(d+e+f) \sqrt{\frac{1}{2}} ; & \text { if } & a, b, c=1 \text { and } d, e, f \neq 1
\end{array}\right.
$$

Theorem 3.3. Let $n, m$ be positive integers, the atom bond connectivity index of a complete bipartite graph denoted by $K_{n, m}$ is

$$
A B C\left(K_{n, m}\right)=\sqrt{n m(n+m-2)} .
$$

Proof. We have mn of the number of edges, each one of them has two vertices that have the same degree, the first vertex of degree $n$ and the second vertex of degree $\mathrm{m}$. Hence by the definition of atom bond connectivity index, we get:

$$
A B C\left(K_{n, m}\right)=n m \sqrt{\frac{n+m-2}{n m}}=\sqrt{n m(n+m-2)} .
$$

Theorem 3.4. Let $k$ be a positive integer, the atom bond connectivity index of a $k$-Bridge graph denoted by $Q\left(a_{1}, a_{2}, a_{3}, \ldots, a_{k}\right)$ is

$$
A B C\left(Q\left(a_{1}, a_{2}, \ldots, a_{k}\right)\right)=\left(a_{1}+a_{2}+\ldots+a_{k}\right) \sqrt{\frac{1}{2}} .
$$

Proof. Let $k=3$ then $G=Q\left(a_{1}, a_{2}, a_{3}\right)$, whose graph is as follows:

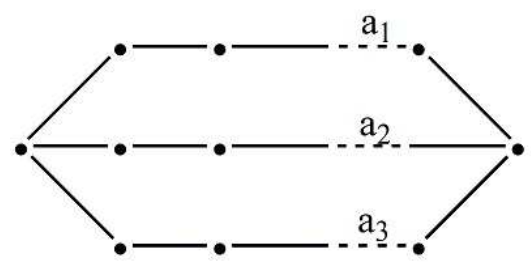

Thus $A B C\left(Q\left(a_{1}, a_{2}, a_{3}\right)\right)=\left(a_{1}+a_{2}+a_{3}\right) \sqrt{\frac{1}{2}}$.

Hence it is true that

$$
A B C\left(Q\left(a_{1}, a_{2}, \ldots, a_{k}\right)\right)=\left(a_{1}+a_{2}+\ldots+a_{k}\right) \sqrt{\frac{1}{2}},
$$


when $k=3$.

Assume that the hypothesis is true for $k=r(r \geq 3)$ that is the ABC index for $Q\left(a_{1}, a_{2}, \ldots, a_{r}\right)$ is given by:

$$
A B C\left(Q\left(a_{1}, a_{2}, \ldots, a_{r}\right)\right)=\left(a_{1}+a_{2}+\ldots+a_{r}\right) \sqrt{\frac{1}{2}} .
$$

Construct the graph $Q_{r+1}$ as follows, $Q\left(a_{1}, a_{2}, \ldots, a_{r}\right)$ has the form

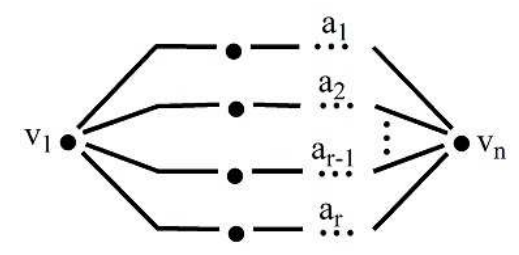

Where $a_{i}$ denotes the position of the edges of graph $Q\left(a_{1}, a_{2}, \ldots, a_{r}\right)$ at the $i^{\text {th }}$ position. The graph $\mathrm{H}$ is the path which contains endings $u_{1}$ and $u_{2}$, and $a_{r+1}$ is the numbers of edges in $\mathrm{H}$ as follows:

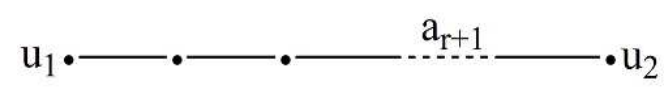

Connect the graph $Q\left(a_{1}, a_{2}, \ldots, a_{r}\right)$ with the graph $\mathrm{H}$ such that $v_{1}=u_{1}$ and $v_{2}=u_{2}$. The vertices $v_{1}=u_{1}$ and $v_{2}=u_{2}$ are of degree $\mathrm{r}+1$, as follows:

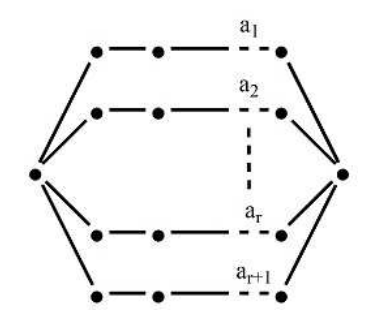

Thus

$$
\begin{aligned}
A B C\left(Q_{r+1}\right) & =A B C\left(Q_{r}\right)+A B C(H) \\
& =\left(a_{1}+a_{2}+\ldots+a_{r}\right) \sqrt{\frac{1}{2}}+a_{r+1} \sqrt{\frac{1}{2}} \\
& =\left(a_{1}+a_{2}+\ldots+a_{r}+a_{r+1}\right) \sqrt{\frac{1}{2}} .
\end{aligned}
$$


Thus hypothesis is true when $k=r+1$.

Hence, it is true when $k=3$. Also as the assumption that it is true for $k=r$, it is shown that it true for $k=r+1$, so we have

$$
A B C\left(Q\left(a_{1}, a_{2}, \ldots, a_{k}\right)\right)=\left(a_{1}+a_{2}+\ldots+a_{k}\right) \sqrt{\frac{1}{2}} .
$$

\section{The ABC Index of Certain Vertex Gluing Graphs}

In this section, we obtain the general formulas for atom bond connectivity index to the vertex gluing of graphs.

Let $K_{4}^{2}$ - homeomorphism be a graph obtained from two deferent $K_{4}-$ homeomorphism graphs $K_{4}\left(a_{1}, a_{2}, a_{3}, a_{4}, a_{5}, a_{6}\right)$ and

$$
K_{4}\left(a_{7}, a_{8}, a_{9}, a_{10}, a_{11}, a_{12}\right),
$$

with common one vertex $v_{1}$ (vertex gluing of graph) (see Fig. 5).

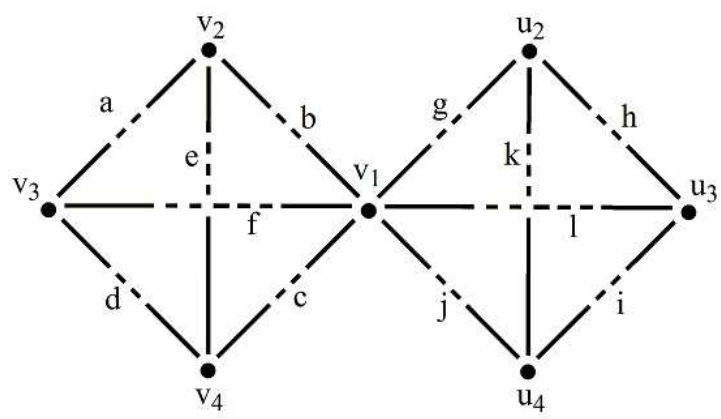

Figure 5: A graph $K_{4}^{2}$ - homeomorphism

Theorem 4.1. Let $a_{i}$ be a positive integer such that $1 \leq i \leq 12$, then atom bond connectivity indices of the $K_{4}^{2}-$ homeomorphism graph are:

1. If $a_{i}=1$, then $A B C\left(a_{i}\right)=\frac{2}{3}, i=1,4,5,8,9,11$.

2. If $a_{i} \geq 2$, then $A B C\left(a_{i}\right)=\sqrt{\frac{1}{2}}, 1 \leq i \leq 12$.

3. If $a_{i}=1$, then $A B C\left(a_{i}\right)=\sqrt{\frac{7}{18}}, i=2,3,6,7,10,12$.

Then $A B C\left(K_{4}^{2}-\right.$ homeomorphism $)=\sum_{i=1}^{12} A B C\left(a_{i}\right)$. 
Proof. We have three cases:

Case 1: If $a_{i}=1$, then $A B C\left(a_{i}\right)=\frac{2}{3}, i=1,4,5,8,9,11$. and any edge $a_{i}$ in this case has two vertices the same degree three, then $A B C\left(a_{i}\right)=\sqrt{\frac{3+3-2}{3.3}}=\frac{2}{3}$.

Case 2. If $a_{i} \geq 2$, then $A B C\left(a_{i}\right)=\sqrt{\frac{1}{2}}, 1 \leq i \leq 12$, and all edges in this case has at least one vertex degree two, then $A B C\left(a_{i}\right)=\sqrt{\frac{1}{2}}, 1 \leq i \leq 12$.

Case 3. If $a_{i}=1$, then $A B C\left(a_{i}\right)=\frac{7}{18}, i=2,3,6,7,10,12$, and all edges in this case it have two vertices, the first one of degree three and the second of degree six, then

$A B C\left(a_{i}\right)=\sqrt{\frac{7}{18}}, i=2,3,6,7,10,12$. Thus $A B C\left(K_{4}^{2}-\right.$ homeomorphism $)=\sum_{i=1}^{12} A B C\left(a_{i}\right)$.

Let $v_{1}-$ gluing of Complete Bipartite graph be a graph obtained from two different Complete Bipartite graphs $K_{n, m}$ and $K_{r, s}$ with common one vertex $v_{1}$ denoted by $K_{n, m}^{r, s}\left(v_{1}\right)$ (vertex gluing of graph),(see Fig.6).

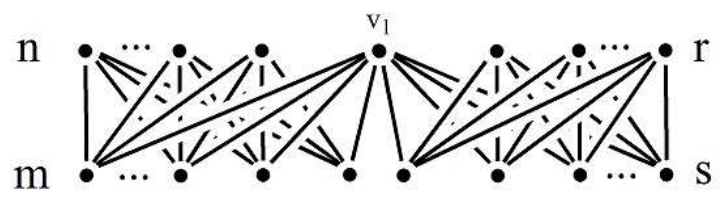

Figure 6: $v_{1}-$ gluing of Complete Bipartite graph $K_{n, m}^{r, s}\left(v_{1}\right)$

Theorem 4.2. Let $n, m, r$ and s be positive integers. Then, the atom bond connectivity index of the $v_{1}-$ gluing of Complete Bipartite graph $K_{n, m}^{r, s}\left(v_{1}\right)$ is

$$
\begin{aligned}
A B C\left(K_{n, m}^{r, s}\left(v_{1}\right)=m(n-1)\right. & \sqrt{\frac{n+m-2}{n . m}}+m \sqrt{\frac{n+m+s-2}{n(m+s)}} \\
& +s \sqrt{\frac{m+r+s-2}{r(m+s)}}+s(r-1) \sqrt{\frac{r+s-2}{r . s}} .
\end{aligned}
$$

Proof. We have two cases: 
Case 1. In complete Bipartite graph $K_{n, m}$ there are nm edges. $m(n-1)$ of them are incident on two vertices of degree $n$ and $m$. The remaining $\mathrm{m}$ are incident on two vertices of degrees $n$ and $(m+s)$.

Case 2. In complete Bipartite graph $K_{r, s}$ there are rs edges. $s(r-1)$ of them incident on two vertices of degree $r$ and $s$. The remaining $s$ are also incident on two vertices of degrees $r$ and $(m+s)$.

From 1 and 2 we get:

$$
\begin{aligned}
A B C\left(K_{n, m}^{r, s}\left(v_{1}\right)=m(n-1) \sqrt{\frac{n+m-2}{n . m}}+m \sqrt{\frac{n+m+s-2}{n(m+s)}}\right. & \\
& +s \sqrt{\frac{m+r+s-2}{r(m+s)}}+s(r-1) \sqrt{\frac{r+s-2}{r . s}} .
\end{aligned}
$$

Let $v_{1}$ - gluing of $n, m-$ Bridge graph be a graph obtained from two different $k-$ Bridge graphs $Q_{1}$ and $Q_{2}$ with one common vertex $v_{1}$ denoted by $Q_{n}^{m}\left(v_{1}\right)$ (vertex gluing of graph),(see Fig.7).

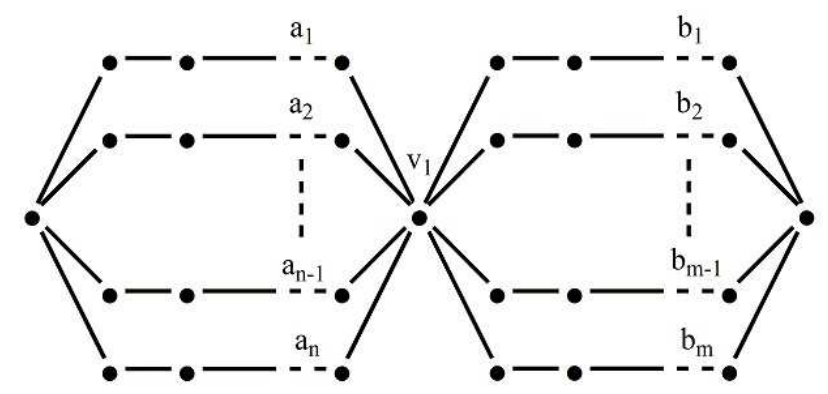

Figure 7: $v_{1}-$ gluing of $n, m-$ Bridge graph $Q_{n}^{m}\left(v_{1}\right)$

Theorem 4.3. Let $n, m$, and $s$ be positive integers. Then, the atom bond connectivity index of the $v_{1}$-gluing of $n, m$ - bridge graph $Q_{n}^{m}\left(v_{1}\right)$ is

$$
A B C\left(Q_{n}^{m}\left(v_{1}\right)\right)=\sum_{i=1}^{n} a_{i} \frac{1}{\sqrt{2}}+\sum_{j=1}^{m} b_{j} \frac{1}{\sqrt{2}} .
$$


Proof. We have $a_{i}, i=1,2,3, \ldots, n$ and $b_{j}, j=1,2,3, \ldots, m$, the numbers of edges, all of them have at least one vertex of degree two, then

$$
A B C\left(Q_{n}^{m}\left(v_{1}\right)\right)=\sum_{i=1}^{n} a_{i} \frac{1}{\sqrt{2}}+\sum_{j=1}^{m} b_{j} \frac{1}{\sqrt{2}}
$$

Let $v_{1}-$ gluing of Web graph be a graph obtained from two different Web graphs $W e b(n, r)$ and $W e b(m, s)$ with one common vertex $v_{1}$ denoted by $W_{n, r}^{m, s}\left(v_{1}\right)$ (vertex gluing of graph),(see Fig.8).

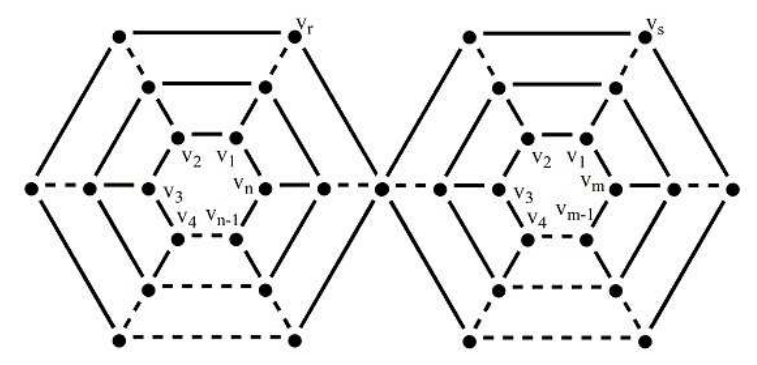

Figure 8: $v_{1}-$ gluing of Web graph $W_{n, r}^{m, s}\left(v_{1}\right)$.

Theorem 4.4. Let $n, r, m$ and $s$ be positive integers. Then, the atom bond connectivity index of the $v_{1}-$ gluing of Web graph $W_{n, r}^{m, s}\left(v_{1}\right)$ is

$$
\begin{gathered}
A B C\left(W_{n, r}^{m, s}\left(v_{1}\right)\right)= \\
\left\{\begin{array}{llc}
2(n+m-2)+6 \sqrt{\frac{7}{18}} ; & \text { if } \quad r, s=2 \\
\frac{2}{3}(3 n+2 m-5)+\frac{\sqrt{6}}{4} m(2 s-5)+\sqrt{\frac{5}{12}}(2 m-1)+\sqrt{\frac{1}{3}}+5 \sqrt{\frac{7}{18}} ; & \text { if } & r=2 \\
\frac{4}{3}(n+m-2)+\frac{\sqrt{6}}{4}(n(2 r-5)+m(2 s-5))+\sqrt{\frac{5}{3}}(n+m-1)+\frac{2}{\sqrt{3}}+4 \sqrt{\frac{7}{18}} ; & \text { if } & r, s \neq 2
\end{array}\right.
\end{gathered}
$$

Proof. We have three cases:

Case 1. If $r, s=2$ then, we have $3(n+m)$ edges of which $3(n+m-2)$ of them are incident on a vertex of degree three. The remaining six edges are incident on a vertex of degree three and a vertex of degree six.

Hence by the definition of atom bond connectivity index, we get:

$$
A B C\left(W_{n, 2}^{m, 2}\left(v_{1}\right)\right)=2(n+m-2)+6 \sqrt{\frac{7}{18}} \quad \text { if } r, s=2,
$$


Case 2. If $r=2$ then, we have $s+2$ cycles containing $2 n+s m$ edges and $n+m(s-1)$ of the edges link the vertices of cycles. Therefore, we have $3 n+m(2 s-1)$ of edges [one of them incident on a vertex of degree four and a vertex of degree six, five of them incident on a vertex of degree three and a vertex of degree six, $3 n+2 m-5$ of them incident on a vertex of degree three, $2 m-1$ of them incident on a vertex of degree three and a vertex of degree four, and finally $m(2 s-5)$ of them incident on a vertex of degree four].

Hence by the definition of atom bond connectivity index, we get:

$$
\begin{aligned}
A B C\left(W_{n, 2}^{m, s}\left(v_{1}\right)\right)=\frac{2}{3}(3 n+2 m-5)+\frac{\sqrt{6}}{4} m(2 s-5)+\sqrt{\frac{5}{12}}(2 m-1) & \\
& +\sqrt{\frac{1}{3}}+5 \sqrt{\frac{7}{18}} .
\end{aligned}
$$

Case 3. If $r, s \neq 2$ then, we have we have $r+s$ of cycles containing $r n+s m$ edges and $n(r-1)+m(s-1)$ of the edges link the vertices of cycles. Therefore, we have $n(2 r-1)+m(2 s-1)$ of edges [two of them incident on a vertex of degree four and a vertex of degree six, four of them incident on a vertex of degree three and a vertex of degree six, $2(n+m-2)$ of them incident on a vertex of degree three, $2(n+m-1)$ of them incident on a vertex of degree three and a vertex of degree four, and finally $n(2 r-5)+m(2 s-5)$ of them incident on a vertex of degree four].

Hence by the definition of atom bond connectivity index, we get:

$$
\begin{aligned}
A B C\left(W_{n, r}^{m, s}\left(v_{1}\right)\right)=\frac{4}{3}(n+m-2)+\frac{\sqrt{6}}{4}(n(2 r-5)+m(2 s-5)) & +\sqrt{\frac{5}{3}}(n+m-1) \\
& +\frac{2}{\sqrt{3}}+4 \sqrt{\frac{7}{18}} .
\end{aligned}
$$

\section{Conclusion}

In this paper, we introduced the general formula for atom bond connectivity index of certain graphs $K_{4}$ - homeomorphism, complete bipartite, k-Bridge graphs and vertex gluing of graphs. 


\section{References}

[1] E. Estrada, Atom-bond connectivity and the energetic of branched alkanes, Chemical Physics Letters, 463 (2008), 422425, http://dx.doi.org/10.1016/j.cplett.2008.08.074.

[2] E. Estrada, L. Torres, L. Rodrguez and I. Gutman, An atom-bondconnectivity index: modelling the enthalpy of formation of alkanes, Indian Journal of Chemistry A, vol. 37, no. 10, pp. 849855, 1998.

[3] B. Furtula, A. Graovac, and D. Vukiević,Atom-bond connectivity index of trees, Discrete Applied Mathematics, vol. 157, no.13, pp. 28282835, 2009. http://dx.doi.org/10.1016/j.dam.2009.03.004.

[4] B. Zhou and R. Xing, On atom-bond connectivity index,Zeitschrift fur Naturforschung A, vol. 66, no. 1-2, pp. 6166, 2011. http://dx.doi.org/10.1515/zna-2011-1-210.

[5] J. Chen and X. Guo, Extreme atom-bond connectivity index of graphs, MATCH Communications in Mathematical and in Computer Chemistry, vol. 65, no. 3, pp. 713722, 2011.

[6] W. Lin, T. Gao, Q. Chen and X. Lin, On The Minimal ABC Index of Connected Graphs with Given Degree Sequence, MATCH Communications in Mathematical and in Computer Chemistry,vol. 69, no. 8, pp. 571578, 2013.

[7] M. V. Diudea, I. Gutman, L. Jntschi, Molecular Topology, Nova, Huntington, 2002.

[8] M. Randić, On characterization of molecular branching, J. Am. Chem. Soc. 97 (1975) 6609 6615. http://dx.doi.org/10.1021/ja00856a001.

[9] A. Behtoei, M. Jannesari, B. Taeri, Maximum Zagreb index, minimum hyperWiener index and graph connectivity, Appl. Math. Lett. 22 (2009) 15711576. http://dx.doi.org/10.1016/j.aml.2009.05.001.

[10] K. C. Das, I. Gutman, B. Furtula, Survey on GeometricArithmetic indices of graphs, MATCH Commun. Math. Comput. Chem. 65 (2011) 595644.

[11] B. Liu, I. Gutman, On general Randic indices, MATCH Commun. Math. Comput. Chem. 58 (2007) 147154.

[12] B. Liu, I. Gutman, On a conjecture on Randic indices, MATCH Commun. Math. Comput. Chem. 62 (2009) 143154.

[13] B. Liu, Z. You, A survey on comparing Zagreb indices, MATCH Commun. Math. Comput. Chem. 65 (2011) 581593.

[14] B. Zhou, N. Trinajstic, Further results on atombond connectivity index of trees, Chem. Phys. Lett. 455 (2008) 120123. http://dx.doi.org/10.1016/j.cplett.2008.02.064.

[15] M. Zhang, B. Liu, On a conjecture about the Randic index and diameter, MATCH Commun. Math. Comput. Chem. 64 (2010) 433442. 
